\title{
Ações de eficiência energética no setor industrial do Brasil
}

\author{
Energy efficiency actions in Brazil's industrial sector
}
Acciones de rendimiento energético en el sector industrial brasileño

\section{Actions d'efficacité énergétique dans le secteur industriel au Brésil}

Francisca Lívia Costa

Pires

francisca_livia@yahoo.com.br Instituto Federal de

Educação, Ciência e

Tecnologia do Ceará - IFCE

Raylla Silveira Souto raylla.ssilveira@hotmail.com Universidade Estadual do Ceará - Uece

Paulo Henrique Pereira Silva paulo.henrique1402@unifor.br Universidade de Fortaleza Unifor

\section{Resumo}

O presente trabalho trata-se de um artigo de revisão, que busca realizar um levantamento de práticas de eficiência energética no setor industrial brasileiro.Para isso, buscaram-se na literatura especializada publicações de até cinco anos relacionada à temática abordada, com intuito de realizar um mapeamento no cenário nacional de programas, políticas e ações de eficiência energética, principalmente no setor industrial. Como base de dados de periódicos para a busca das publicações, foram usados Web of Science, Science Direct e IEEE Xplore. Inicialmente, as buscas retornaram 369 artigos publicados; destes, apenas 351 foram possíveis ser acessados. Verificou-se também que alguns artigos se tratavam de publicações repetidas, e, após a exclusão destes, restaram 326 artigos para a análise. Destes, apenas 75 apresentaram conteúdo de estudo de caso, projeto ou levantamento bibliográfico com relação à temática pesquisada. Por fim, apenas 19 publicações foram selecionadas para análise de dados deste estudo. Entre os dados analisados, encontram-se os anos das publicações selecionadas, a porcentagem das publicações por setor, o número de publicações por regiões, os principais aspectos de execução dos trabalhos, as metodologias aplicadas e os principais temas abordados em cada um dos artigos. Assim, pôde-se concluir que alguns setores industriais já desenvolvem pesquisas que buscam melhorar os aspectos que envolvem a eficiência energética no ambiente industrial, tendo em vista que as indústrias são responsáveis por grande parte do consumo de energia no país, sendo essas iniciativas imprescindíveis para se buscar cada vez mais um consumo racional das fontes energéticas disponíveis no país.

Palavras-chave: Eficiência Energética.Setor industrial. Revisão de literatura.

\begin{abstract}
This article is a basic research about energy efficiency practices in the Brazilian industrial sector, for that, we searched in the specialized literature publications from the last five years related to the topic addressed, to carry out mapping in the scenario of energy efficiency programs, policies, and actions, mainly in the industrial sector. As a database to search for publications, the journals used were the Web of Science, Science Direct, and IEEE Xplore. Initially, searches returned 369 published articles, of which only 351 were accessible, it was also found that some articles were repeated publications, and after excluding them, 326 articles remained for analysis. Of these, only 75 presented the content of a case study, Project, or bibliographic survey, concerning their searched theme. Finally, only 19 publications were selected for data analysis in this study, among the analyzed data are the years of selected publications, percentage of publications by sector, number of publications by regions, the main aspects of the work, applied methodologies and the main themes covered in each of the articles. Thus, it can be concluded that some industrial sectors are already carrying
\end{abstract}


out research that seeks to improve aspects involving energy efficiency in the industrial environment, bearing in mind that industries are responsible for a large part of energy consumption in the country, and these initiatives are impressive. To seek more and more rational consumption of energy sources available in the country.

Keywords: Energy efficiency. Industrial sector. Literature revision.

\section{Resumen}

El presente trabajo se trata de un artículo de revisión, que busca realizar una búsqueda de prácticas de rendimiento energético en el sector industrial brasileño. Para tanto, se buscó en la literatura especializada publicaciones de hasta cinco años relacionadas con el tema propuesto, con la intención de realizar un mapeo del escenario nacional de programas, políticas y acciones de rendimiento energético, principalmente en el sector industrial. Como base de datos para la búsqueda de las publicaciones fueron utilizados Web of Science, Science Direct y IEEE Xplore.Inicialmente, las búsquedas encontraron 369 artículos publicados; de estos solo 351 fueron posibles obtener acceso.Se verificó también que algunos artículos eran publicaciones repetidas, y, después de la exclusión de estos, restaron 326 artículos para el análisis. De estos, solo 75 presentaron contenido de estudio de caso, proyecto o búsqueda bibliográfica con relación a la temática investigada. Por fin, solamente 19 publicaciones fueron seleccionadas para análisis de datos de este estudio. Entre los datos analizados, se encuentran los años de las publicaciones seleccionadas, el porcentaje de las publicaciones por sector, el número de publicaciones por regiones, los principales rasgos de ejecución de los trabajos, las metodologías aplicadas y los principales temas tratados en cada uno de los artículos. Se puede, entonces, concluir que algunos sectores industriales ya desarrollan investigaciones que buscan mejorar los aspectos que implican el rendimiento energético en el ambiente industrial, teniendo en cuenta que las industrias son responsables por gran parte del consumo de energía en el país, siendo estas iniciativas imprescindibles para buscar cada vez más un consumo racional de las fuentes energéticas disponibles en el país.

Palabras-clave: Rendimiento energético. Sector industrial. Revisión de literatura.

\section{Résumé}

Ce travail est un article de revue qui cherche à étudier les pratiques d'efficacité énergétique dans le secteur industriel brésilien. À cette fin, des publications liées au sujet ont été recherchées dans la littérature spécialisée (elles devraient avoir 5 ans ou moins), afin de cartographier le scénario national des programmes, des politiques et des actions d'efficacité énergétique, principalement dans le secteur industriel. En tant que base de données pour rechercher des publications, Web of Science, Science Direct et IEEE Xplore ont été utilisés. Au départ, les recherches ont renvoyé 369 articles publiés ; parmi eux, on n'a eu accès qu'à 351 . On a aussi constaté que certains articles étaient répétés et, après leur exclusion, 326 articles ont resté à analyser. Parmi ceux-ci, seulement 75 présentaient le contenu d'une étude de cas, d'un projet ou d'une enquête bibliographique concernant le thème recherché. Enfin, seulement 19 publications ont été sélectionnées pour l'analyse des données dans cette étude. Parmi les données analysées, il y a les années des publications sélectionnées, le pourcentage de publications par secteur, le nombre de publications par régions, les principaux aspects de l'exécution des travaux, les méthodologies appliquées et les principaux thèmes abordés dans chacun des articles. Ainsi, on a pu conclure que certains secteurs industriels mènent déjà des recherches qui visent à améliorer les aspects liés à l'efficacité énergétique dans l'environnement industriel. Cela est vraiment important car les industries sont responsables d'une grande partie de la consommation d'énergie dans le pays, et ces initiatives sont essentielles pour rechercher une consommation de plus en plus rationnelle des sources d'énergie disponibles dans le pays.

Mots-clés: Efficacité énergétique Secteur Secondaire Revue de Littérature 


\section{Introdução}

A demanda por energia tem aumentado exponencialmente nas últimas décadas, principalmente por conta de fatores como o aumento do consumo dos recursos naturais, que tem registrado índices elevados, especialmente por conta do crescimento populacional. Esse cenário tem sido um dos principais temas de discussões em fóruns, conferências e congressos relacionados à eficiência energética, meio ambiente e sustentabilidade (GOLDEMBERG e LUCON 2007; FORTES et al., 2017).

Os recursos energéticos são imprescindíveis para o desenvolvimento dos países. E, atrelado a esse fato, existem consequências que afetam o meio ambiente, como aumento na concentração de gases de efeito estufa (GEE), especialmente o dióxido de carbono $\left(\mathrm{CO}_{2}\right)$, que intensifica o efeito estufa e o aquecimento decorrente dele. Esse fato consta no relatório do IPCC (Painel Intergovernamental sobre Mudanças Climáticas) do ano 2007, no qual se confirma que as causas do aquecimento global são conferidas, em maior parte, às ações humanas, principalmente às atividades relacionadas à emissão do $\mathrm{CO}_{2}$ (CAMIOTO, REBELATTO, ROCHA, 2016).

Segundo a Empresa de Pesquisa Energética (2019), aproximadamente 81,1\% da matriz energética mundial é baseada na exploração de fontes de energias fósseis,tais como carvão (27,1\%), petróleo e derivados $(31,9 \%)$ e gás natural $(22,1 \%)$. Essas fontes são as principais responsáveis pelo aumento das emissões de $\mathrm{CO}_{2}$ na atmosfera, além do fato que as reservas dessas fontes são finitas. Nesse sentido, países como Estados Unidos, que consomem, por ano, um quarto da energia produzida no mundo, e o Canadá, que apresenta o maior consumo de energia per capita, juntaram-se a países como China, Brasil e Índia, cujo consumo de energia está a aumentar ano após ano. Considerando que, quanto maior o nível de atividade econômica, maior é uso de energia e maiores são impactos ambientais causados, necessita-se que as nações criem políticas voltadas à eficiência energética, visando a adoção de recursos de fontes renováveis para diminuir o uso das fontes fósseis, diminuindo os impactos ao meio ambiente (CAMIOTO, REBELATTO, ROCHA, 2016).

Apesar de o Brasil ter apresentado um aumento na demanda por energia, sua matriz energética é composta, em sua maior parte, por fontes de energias renováveis, mais do que no resto do mundo. Segundo estimativa da Empresa de Pesquisa Energética (EPE), em 2019, somando lenha e carvão vegetal, hidráulica e derivados de cana de açúcar, as fontes de energias renováveis totalizaram $42,9 \%$, quase a metade da matriz energética nacional.

No Brasil o setor que mais consome recursos energéticos são as indústrias, pois, além delas consumirem boa parcela da energia elétrica produzida no país, fabricam bens que consomem energia (SOLA e KOVALESKI, 2004). De acordo com a Confederação Nacional da Indústria (CNI), as indústrias são responsáveis pelo consumo de, pelo menos, $42 \%$ da energia elétrica do Brasil, tendo em vista que,aproximadamente, 537 mil unidades estão em pleno funcionamento em todo território brasileiro. Em relação à eficiência energética, o Conselho Americano para uma Economia eficiente em energia (ACEEE, sigla em inglês), realizou uma pesquisa em que o Brasil ficou em $15^{\circ}$ lugar entre as dezesseis maiores economias do mundo. Nos critérios de análise do estudo, o qual tem a Alemanha como a nação mais eficiente no cenário mundial, o pior desempenho do Brasil foi relacionado ao setor industrial, recebendo apenas 2 dentre os 25 pontos possíveis. Dessa forma, fica evidente a importância entre setor industrial e a importância da adoção de políticas em eficiência energética para o setor (FLUXO CONSULTORIA, 2017).

Diante dos fatos citados, este trabalho tem como objetivo fazer uma revisão na literatura especializada de artigos científicos que apontam ações de eficiência energética desenvolvidas pelo setor industrial brasileiro.

\section{Metodologia}

O presente trabalho pode ser classificado como pesquisa bibliográfica, do tipo revisão integrativa de literatura, uma vez que se trata da análise técnica de diversos trabalhos já executados e publicados, apresentando uma abordagem qualitativa do tema. Em relação aos objetivos, identifica-se como uma pesquisa exploratória, apresentando a síntese de um levantamento bibliográfico sobre o impacto da eficiência energética na indústria brasileira. 
Com a metodologia utilizada, foi apresentado um mapeamento das ações de eficiência energética, realizadas e publicadas, no setor industrial do Brasil. Para isso, foi executada uma pesquisa inicial sobre o cenário nacional de programas, políticas e ações sobre a temática. Em seguida, foram definidas as palavraschaves para aprofundamento da pesquisa bibliográfica. Foram selecionados, então, dezenove artigos publicados para análise e comparação dos resultados.

Para a realização da busca por artigos científicos, foram consideradas as seguintes bases de periódicos: Web of Science, Science Direct e IEEE Xplore. Outras bases foram consideradas, inicialmente, porém não retornaram uma quantidade significativa de publicações.

Com o objetivo de tornar a pesquisa o mais abrangente possível, foram escolhidos três termos como palavraschave: eficiência energética, indústria e Brasil. Para ampliar ainda mais os resultados obtidos, aplicouse uma expressão lógica formada a partir das palavras-chave na busca avançada das bases. Assim, a expressão lógica de busca, aplicada na língua inglesa e considerando os últimos cinco anos, foi: (Energy efficiency OR energyconservation) AND (industry OR industrial OR industries) AND (Brazil OR brazilian).

Conforme mostrado na Fig. 1, a pesquisa inicial retornou 369 artigos publicados. Destes, puderam ser acessados e arquivados 351 publicações. Como as bases, algumas vezes, realizam a pesquisa nas mesmas revistas, alguns trabalhos repetidos foram excluídos, restando 326 artigos para análise. Destes, apenas 75 apresentaram estudos de caso, projetos ou levantamentos bibliográficos com alguma relação ao tema pesquisado.

Como o objetivo do trabalho foi analisar aplicações de medidas de eficiência energética para a redução do consumo de energia elétrica, apenas 19 publicações puderam ser consideradas para análise. Os demais trabalhos foram excluídos por apresentar: medidas de eficiência relacionadas apenas ao calor, discussões sobre políticas públicas e regulamentações, outras revisões bibliográficas e ações realizadas fora do Brasil.

Figura 1 - Resultado da metodologia de pesquisa.

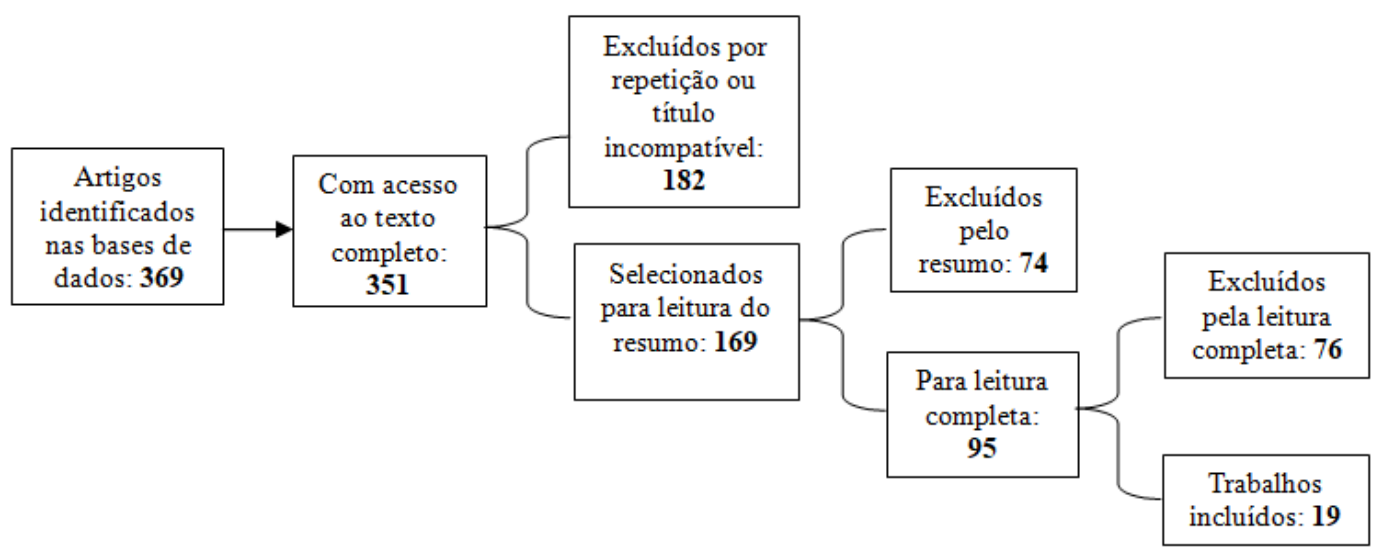

Fonte: Elaborado pelos autores (2020).

\section{Revisão bibliográfica}

Com a aplicação da metodologia descrita acima, foram selecionados dezenove trabalhos publicados em periódicos para discussão. Os trabalhos escolhidos abordam diferentes temas relacionados à eficiência energética e, em sua maioria, apontam para a necessidade de mais regulamentações e maior conscientização dos gestores das indústrias. Indicam, também, ótimos resultados em redução de consumo de energia elétrica com a aplicação de algumas medidas de eficiência.

Em estudo realizado por Da Silva, Oliveira e Souza (2019) é proposto um método de modelagem para previsão do consumo de energia elétrica, em longo prazo, no setor industrial de celulose e papel, que permite a introdução de medidas de eficiência energética nos cálculos. O trabalho apresenta a previsão de elevação 
Ações de eficiência energética no setor industrial do Brasil

no consumo até 2050 , com os percentuais de redução que podem ser atingidos com a aplicação de dezesseis ações de eficiência energética nos sistemas de ventilação, ar comprimido, refrigeração, motores em geral e iluminação. No cenário em que não se considera a eficiência energética, o modelo proposto previu que o consumo de eletricidade aumentará 35,73\% entre 2016 e 2030 e 107,43\% entre 2016 e 2050 . Porém, quando são aplicadas medidas de eficiência, o consumo de eletricidade deve apresentar um crescimento de 10,04\% entre 2016 e 2030 e 56,82\% entre 2016 e 2050.

Em pesquisa realizada por Dos Santos e Spinelli (2019) é realizada a análise do uso de um gerador termoelétrico na recuperação de energia residual do processo de lingotamento contínuo de aços. Para as indústrias do aço, torna-se importantíssima a conservação de energia, uma vez que requer uma grande quantidade de recursos e, nesse sentido, a recuperação de calor é um método de conservação de energia de grande relevância. A proposta deste trabalho é estimar, através de simulação matemática e métodos de ajuste, o aproveitamento do calor residual a partir de módulos termoelétricos reversíveis.

Pontes, Yamauchi e Silva (2019) apresentam um estudo sobre as torres de resfriamento para água utilizada em indústrias químicas. No trabalho, um modelo matemático é aplicado para a simulação de resfriamento, considerando o clima de quatro cidades brasileiras: Manaus, Salvador, São Paulo e Porto Alegre. Com base nas simulações, são propostas estratégias para a operação do ventilador da torre com o objetivo de reduzir o consumo de energia elétrica. Essa redução se mostra interessante, uma vez que o resfriamento de água exige um consumo de energia elétrica consideravelmente alto para motores de ventiladores e bombas da torre de resfriamento. Entre as estratégias apresentadas para redução do consumo nas torres estão: a folga da torre de resfriamento, torres de resfriamento com várias células, ajuste da temperatura da água de resfriamento e parâmetros operacionais da torre de resfriamento em função da temperatura do ar ambiente.

Moraes, Machado e Silva (2019) avaliaram o impacto de um programa de eficiência energética em um fabricante multinacional de eletrodomésticos com sede em Curitiba - Paraná e São Carlos - São Paulo. Após diversas ações de eficiência energética serem aplicadas no ano 2017, uma análise de desempenho energético foi realizada e verificou-se uma redução na filial de Curitiba de $1233 \mathrm{MWh}(2,22 \%)$ durante o ano, enquanto a redução na planta de São Carlos foi de $1005 \mathrm{MWh}$ (2,57\%) para o mesmo ano. De acordo com os autores, se todo o setor industrial reduzisse seu consumo de energia na mesma quantidade conseguida com o programa de sustentabilidade aplicado, a redução total do consumo de energia seria cerca de 3,3 TWh por ano.

No trabalho realizado por Birru, Erlich e Martin (2019) foram analisados parâmetros operacionais relacionados à energia na indústria da cana-de-açúcar, com o objetivo de identificar melhorias na eficiência energética e no potencial de exportação de excedente de eletricidade. As modificações consideradas nas usinas incluíram: caso 1- introdução de motores e turbinas de compressão de maior capacidade; caso 2 - o fluxo total de bagaço é considerado para obter o fluxo máximo de vapor e, nesse cenário, os moinhos considerados são os mesmos do caso 1; caso 3 - a secagem do bagaço resulta em menor teor de umidade do bagaço, o que melhora a temperatura de combustão na caldeira, melhorando a relação vapor/bagaço melhora. A análise técnica e econômica foi realizada em indústrias de nove localidades brasileiras. Concluiu-se, no trabalho, que investimentos com alto custo, como a instalação de caldeiras de alta pressão, nem sempre podem ser a modificação necessária que precisa ser feita se for necessária uma exportação de energia excedente. Outras opções mais baratas, como secagem de bagaço e substituição de acionamentos mecânicos a vapor por elétricas, podem ser introduzidas. Além disso, a modernização da unidade de cogeração de uma usina de açúcar para fins de produção de excedente de energia nem sempre é viável devido a, entre outros, a sazonalidade da produção de açúcar e os custos mais altos associados aos equipamentos modernos.

No estudo de Mascarenhas et al. (2019) são considerados os conceitos de energia, exergia e sustentabilidade, aplicados a compressores de ar industrial. O diferencial desse trabalho consiste em considerar vários indicadores de sustentabilidade, como número de esgotamento, índice de sustentabilidade, índice de exergia de resíduos e fator de efeito ambiental. Além disso, são apresentadas diversas medidas de eficiência energética e suaviabilidade econômica. Assim, para o desenvolvimento do trabalho, foram realizadas as 
análises: energética, de exergia, de sustentabilidade e de economia de energia, de mitigação da emissão de dióxido de carbono e econômica para o sistema de compressão, utilizando como ferramenta o software de simulação RETScreen. Considerou-se, para o estudo de caso, uma indústria química localizada na Bahia. Para a simulação foram consideradas as especificações dos compressores de ar e medições de temperatura, pressão, consumo de energia, entre outras. Diversas ações foram propostas, tais como: redução da pressão de descarga, substituição de compressores e instalação de motores eficientes. Este trabalho demonstrou que análises de exergia e sustentabilidade podem ser aplicadas para identificar compressores ineficientes, auxiliando nas tomadas de decisão no ambiente industrial. Também demonstrou que a análise de entalpia e energia é ineficaz na avaliação da saída do trabalho mecânico do ar comprimido.

Em pesquisas realizadas por Sola e Mota (2019) foram feitas avaliações dos fatores que influenciam a aplicação do gerenciamento de energia em indústrias, considerando diferentes perspectivas, tais como: economia, contingência, mudança tecnológica e comportamental. A metodologia utilizada foi a aplicação de um questionário, considerando quarenta variáveis a diversos setores industriais. Após análise estatística, as variáveis foram reduzidas a oito fatores relevantes que influenciam na aplicação do gerenciamento de energia. Neste estudo, quarenta variáveis ligadas ao gerenciamento de energia foram analisadas, sendo reduzidas para oito fatores relevantes: estratégicos, motivacionais, produtivos, operacionais, econômicos, de suporte, de competitividade e ambientais. Observou-se que aspectos contingenciais, especialmente relacionados ao mercado, são decisivos para a adoção de estratégias, afetando o modelo de gestão de energia adotado pelas empresas. Concluiu-se, também, que o envolvimento da equipe, principalmente os gerentes de nível intermediário, é essencial para desenvolver ações em áreas estratégicas e operacionais na gestão de energia. Observou-se, também, que a área de produção é propulsora de ações em gerenciamento de energia, influenciando os investimentos em eficiência energética.

O trabalho de Zanardo et al. (2018) apresenta um modelo de auditoria energética a ser implantado no setor industrial com o objetivo de identificar o desempenho energético através de diversos indicadores. Através de revisão bibliográfica, foram identificados os fatores mais recorrentes para a composição do índice de eficiência energética de uma instalação. Foram considerados 58 fatores no modelo proposto, estando relacionados às áreas de ações administrativas, motores elétricos, sistemas elétricos, iluminação, sistemas de ar condicionado e sistemas de ar comprimido. O setor industrial brasileiro foi escolhido para avaliação do modelo, o qual apresentou um índice de eficiência energética de $51,45 \%$. Também foram realizadas simulações que mostraram que, com algumas ações específicas, a empresa poderia ter um aumento de $34,59 \%$, sobre esse indicador.

O desenvolvimento de um sistema capaz de minimizar os custos com energia elétrica através de um processo de otimização da programação de produção industrial é apresentado em estudo de Tonini et al. (2018), no qual é considerada a geração distribuída, através de fontes de energia renováveis, as características de um processo produtivo industrial do tipo job shop, a tarifação utilizada por empresas no mercado de energia brasileiro e os custos adicionais relacionados à atividade noturna dos funcionários envolvidos na execução dos processos de produção. O sistema desenvolvido possui uma interface simples e eficiente, que facilita aos usuários a análise dos resultados. O algoritmo calcula a demanda prevista para cada hora, sendo capaz de suprir os processos, verificando se pode ser suprida com a energia renovável e, em seguida, ela é separada por horário de ponta e de fora de ponta e quantificado o número de horas noturnas trabalhadas. Sendo possível, desse modo, possível realizar os cálculos dos custos.

Estudos realizados por Macedo, Mota e Sola (2018) propõem um modelo de decisão multicritério para apoiar um problema de substituição de motores em uma indústria química, atendendo aos padrões mínimos de desempenho energético estabelecidos pela Lei Brasileira de Eficiência Energética. $O$ algoritmo proposto utiliza os seguintes critérios para tomada de decisão: anos de uso, quantas vezes foram rebobinados, quantidade de falhas, importância para a produção, potência nominal do motor, carregamento e valor presente líquido. A partir desses critérios, o modelo elabora um plano de substituição dos motores, considerando restrições orçamentárias e estratégicas da empresa. 
Uma análise de desempenho energético do setor siderúrgico é apresentada em pesquisas realizadas por Silva, Mathias e Bajay (2018). Nesse trabalho, foi considerada uma amostra de cinco usinas integradas com produção interna de coque, que representam mais da metade do aço bruto produzido no Brasil. Foi realizada uma análise de desempenho energético, seguida da avaliação de aplicabilidade de uma série de medidas de eficiência energética, curvas de fornecimento de conservação de energia para combustíveis e eletricidade e, por fim, a avaliação do custo dessas medidas. Foi considerado o histórico detalhado de dados de energia nas usinas ArcelorMittal, CSN, Usiminas Ipatinga, Usiminas Cubatão e Gerdau. Foi avaliado o potencial de eficiência energética a partir de 17 medidas, nos setores de sinterização, produção de coque, produção de ferro, produção de aço, fundição e laminação. O potencial de eficiência estimado para combustíveis foi de 3,02 GJ/tcs (Gigajoule/tonnesofcrudesteel), ou 12\%, e, para a eletricidade, de 1,51GJ/tcs.

Aguiar, Pontes e Ferreira (2018) apresentaram em suas pesquisas a relação custo-benefício dos motores de indução de baixa potência, cuja eficiência foi melhorada após o rebobinamento com enrolamentos eficientes, como opção para a substituição dos motores após o fim de sua vida útil, sendo realizada uma análise técnicoeconômica. Três casos foram estudados: a substituição de um motor da classe IE2 existente por uma unidade IE3 ou IE4; a substituição de um motor IE2 existente por um rebobinado. As eficiências dos motores em cada caso foram determinadas por meio de dois métodos: medições diretas e indiretas. Em todos os casos, as medições foram realizadas em bancada experimental. A análise econômica foi executada, considerando a análise de custo do ciclo de vida e o custo-efetividade dos métodos. Concluiu-se no trabalho que o rebobinamento do motor IE2 existente, substituindo os enrolamentos anteriores para enrolamentos eficientes, é a opção mais econômica para a indústria. $O$ trabalho considerou as características do mercado brasileiro e os seus parâmetros econômicos.

No trabalho realizado por Lima, Ribeiro e Perez (2018) foi feita uma análise sobre a eficiência no uso da energia em indústrias de laticínios no Brasil. A pesquisa também fornece recomendações de políticas ao setor sobre a utilização da energia de maneira mais eficiente, limpa e renovável em médio e longo prazo. O estudo de caso foi realizado em estabelecimentos industriais de queijo, através da aplicação de um questionário on-line a 1.188 estabelecimentos registrados no Ministério da Agricultura, Pecuária e Abastecimento. Métodos estatísticos foram aplicados com o objetivo de caracterizar a amostra, de acordo com seu perfil, uso e gasto dos recursos energéticos e eficiência energética, verificando tendências de concentração de ineficiência energética. Esse estudo também identificou altas ineficiências em relação ao uso de eletricidade e energia térmica nessas indústrias.

O trabalho de Guedes et al. (2017) discute a utilização de barramentos CC em indústrias, de modo a contribuir para a eficiência energética. Uma melhoria na eficiência é conseguida, pois o uso de barramentos CC de distribuição reduz grande parte dos conversores de potência, podendo ser utilizado apenas um retificador bidirecional para atender a um grupo com vários motores, assim como facilita a conexão com fontes renováveis de energia. Ao contrário das microrredes CC tradicionais, são considerados os efeitos das variações de tensão no barramento para um ambiente industrial. A estrutura de conversores, barramento e supercapacitor é proposta, dimensionada e testada experimentalmente.

Para Lacerda et al. (2017), perdas associadas aos processos de conversão podem ser reduzidas com a alimentação das cargas diretamente de um barramento $C C$, associada ao uso de energia fotovoltaica. Para demonstração, realizou-se um estudo de caso, considerando uma planta industrial fictícia. O sistema proposto mostrou-se tecnicamente viável através da análise por simulação, com uma melhoria na eficiência.

Silva, Méxas e Quelhas (2017) apresentam um estudo sobre os fatores que restringem a adoção de tecnologias limpas em indústrias de cerâmica vermelha. Os fatores foram determinados a partir de revisão bibliográfica e questionário aplicado a gestores e trabalhadores nessas indústrias, localizadas em um complexo industrial do Rio de Janeiro. Entre as tecnologias limpas consideradas está a eficiência energética e os fatores restritivos identificados, estavam: fatores institucionais, financeiros/econômicos, tecnológicos e sociais/culturais.

Santana e Bajay (2016) propõe uma nova configuração de políticas de eficiência energética para a indústria brasileira, com base em experiências bem sucedidas em outros países. São destacados no trabalho 
potenciais de economia que podem ser atingidos por meio de políticas, para promover o uso de equipamentos industriais com maior eficiência e para melhorar a eficiência geral por meio do gerenciamento de energia. Entre as propostas apresentadas, estão: a formulação de uma política pública ampla e de longo prazo, a criação de uma agência executiva e centros de avaliação industrial, incentivos fiscais e de crédito, metas de economia de energia com procedimentos de medição e verificação, incentivos fiscais para a adoção de tecnologias eficientes e o aprimoramento das atuais políticas informativas.

Sauer et al. (2015) apresentam uma visão geral dos motores instalados no setor industrial brasileiro e avaliam o impacto, para o consumo de energia, caso uma regulamentação com critérios mínimos de eficiência energética seja implantada. Os ensaios experimentais, realizados em uma amostra com 276 modelos diferentes de motores elétricos, apresentaram, na maioria dos casos, índices de eficiência muito abaixo dos valores da regulamentação brasileira para motores de alto desempenho.

Da Silva et al. (2015) aplicam um modelo estatístico para prever a demanda por energia elétrica pelo setor de celulose e papel, no Brasil, até o ano de 2035, considerando diferentes cenários de eficiência energética. Foram considerados quatro cenários para a análise: (a) nenhuma medida de eficiência energética foi aplicada, (b) algumas medidas de eficiência foram consideradas, (c) mais medidas de eficiência consideradas e (d) todas as medidas possíveis são aplicadas e a redução no consumo é máxima. Obtendo, através do modelo, previsões de crescimento de consumo de energia elétrica de 2020 a 2035 de: (a) $69 \%$, (b) $55 \%$, (c) $23 \%$ e (d) $5 \%$.

\section{Análise dos trabalhos selecionados}

Sobre os artigos discutidos na seção anterior deste trabalho, são mostradas, a seguir, algumas comparações e observações realizadas a partir das principais informações de cada artigo. Por exemplo, nota-se o crescimento de publicações na área por ano, no período considerado de 2015 a 2019, conforme apresentado na Fig. 2.

Figura 2 - Publicações em periódicos selecionadas por ano.

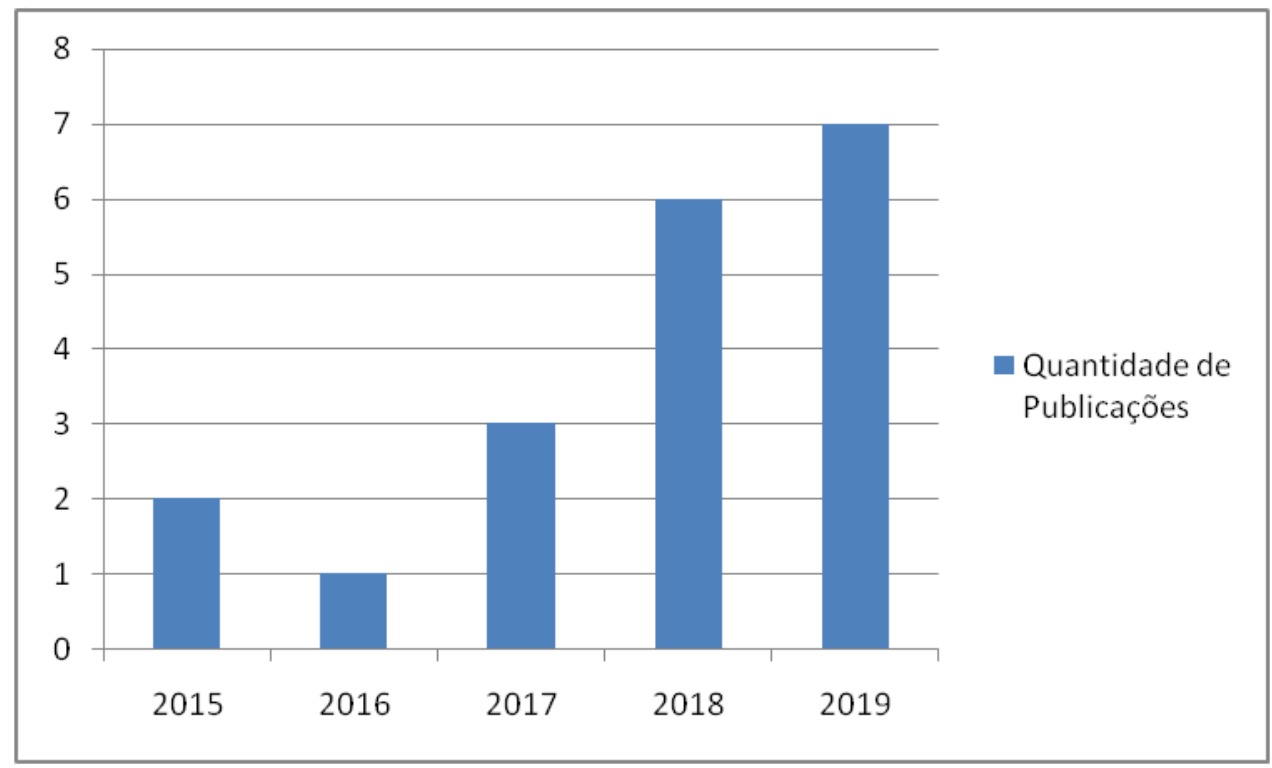

Fonte: Elaborado pelos autores (2020).

Em relação aos setores produtivos, apresenta-se, na Fig. 3, o percentual de trabalhos publicados por setor. Desse gráfico, pode-se ressaltar que a maioria dos artigos se refere a propostas de aplicações genéricas, que podem ser adaptadas a qualquer ambiente industrial. A segunda maior contribuição é das indústrias químicas ( $16 \%$ dos artigos analisados), com a maior parte dos trabalhos relacionados ao aproveitamento de calor dos processos. As áreas com o terceiro maior quantitativo de trabalhos são a de fabricação do aço e a de papel 
(ambas presentes em 11\% dos trabalhos selecionados), em que são apresentadas simulações com a aplicação de diversas ações de eficiência energética.Para os setores de fabricação de eletrodomésticos, usinas de cana de açúcar, automotivo, de laticínios e cerâmica, apenas uma publicação foi selecionada.

Figura 3 - Percentual de publicações científicas por setor industrial.

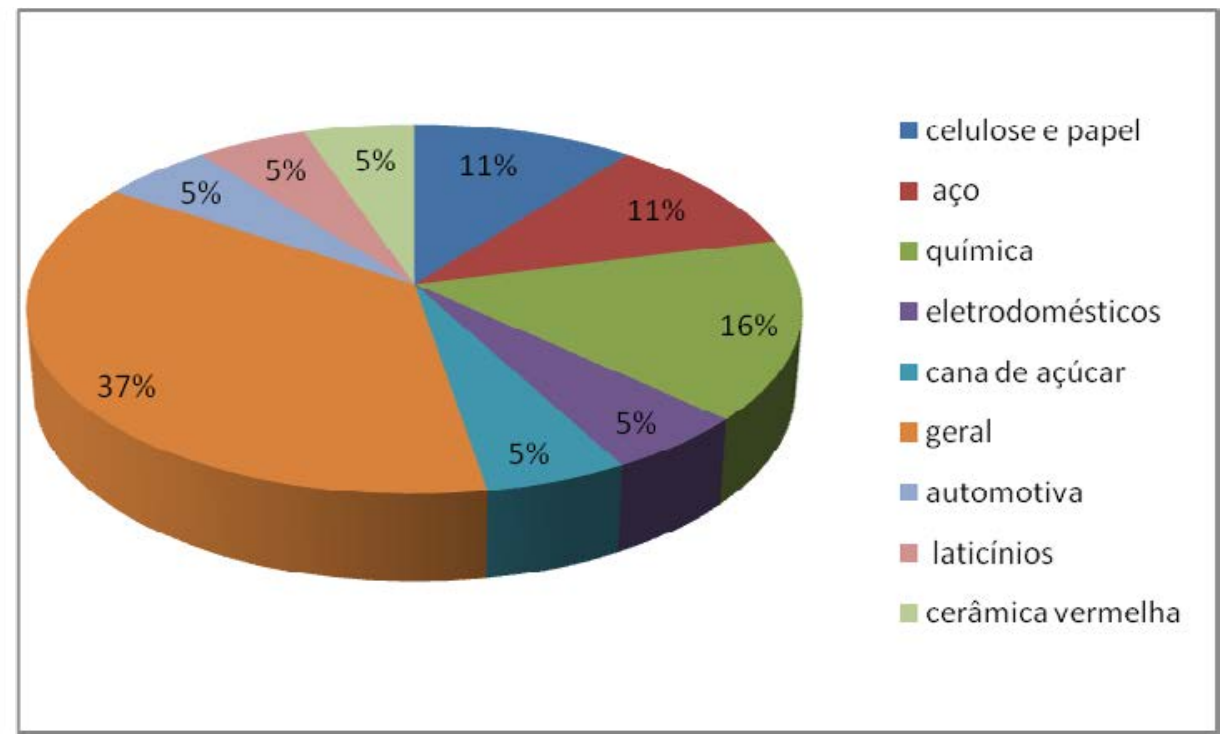

Fonte: Elaborado pelos autores (2020).

$\mathrm{Na}$ Figura 4 são analisadas as localidades de desenvolvimento dos trabalhospublicados. A maioria dos trabalhos discutiu ações de eficiência energética para indústrias no Brasil, sem especificação de local ou abrangendo diversas localidades e regiões. Os demais trabalhos tratam de aplicações práticas ou estudos de caso em uma única localidade ou em uma quantidade limitada de localidades, sendo a maior quantidade de trabalhos (seis) relacionados à região Sudeste do Brasil. Em segundo lugar, indústrias da região Nordeste foram estudadas em cinco publicações e, destacam-se, em menor quantidade, trabalhos desenvolvidos sobre a região Centro-Oeste.

Figura 4 - Aplicação dos trabalhos por região do país.

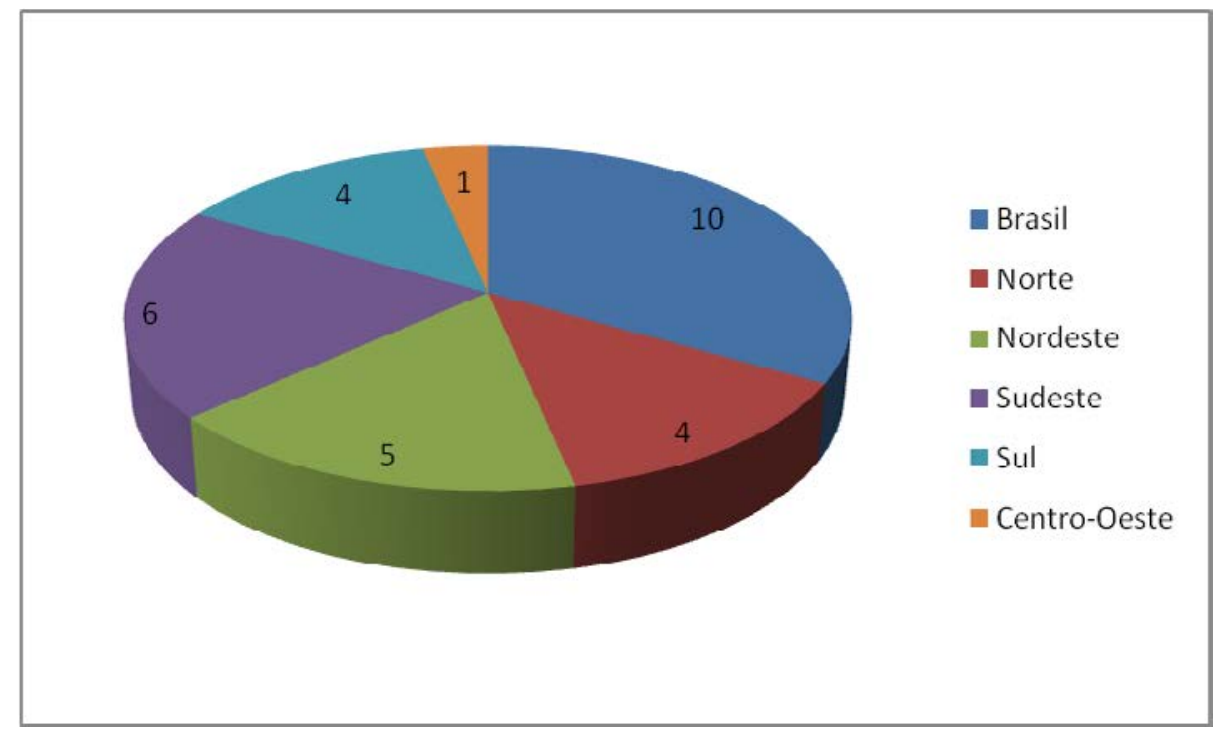

Fonte: Elaborado pelos autores (2020). 
Como pode ser visto na Tab. 1, todos os artigos selecionados descreveram bem os aspectos tecnológicos relacionados à aplicação de determinadas medidas de eficiência energética, apresentando: a redução de consumo, as mudanças nos processos, as trocas de equipamentos, os softwares de simulação e os modelos matemáticos desenvolvidos, entretanto apenas $47 \%$ das publicações analisadas (nove) discutiram sobre a viabilidade econômica das propostas. Desses trabalhos, apenas Pontes, Yamauchi e Silva (2019); Mascarenhas et al. (2019); Birru, Erlich e Martin (2019) e Aguiar, Pontes e Ferreira (2018) apresentaram a análise de viabilidade econômica do projeto desenvolvido. Os demais trabalhos avaliaram a influência dos fatores econômicos em determinados projetos de eficiência, através de revisão bibliográfica ou aplicação de questionários. Ainda menos discutidos são os impactos ambientais das ações propostas. O ponto de vista ambiental é analisado em apenas $26 \%$ das publicações (cinco artigos).

Tabela 1 - Aspectos considerados na execução dos trabalhos publicados.

\begin{tabular}{|c|c|c|c|c|}
\hline Publicação & Tema & $\begin{array}{l}\text { Aspectos } \\
\text { técnicos }\end{array}$ & $\begin{array}{c}\text { Aspectos } \\
\text { econômicos }\end{array}$ & $\begin{array}{c}\text { Aspectos } \\
\text { ambientais }\end{array}$ \\
\hline Da Silva, Oliveira e Souza (2019) & Previsão de consumo & $\checkmark$ & & \\
\hline Dos Santos e Spinelli (2019) & Aproveitamento de calor & $\checkmark$ & & \\
\hline Pontes, Yamauchi e Silva (2019) & Torres de resfriamento & $\checkmark$ & $\checkmark$ & \\
\hline Moraes, Machado e Silva (2019) & Programas de eficiência & $\checkmark$ & & \\
\hline Birru, Erlich e Martin (2019) & Viabilidade econômica & $\checkmark$ & $\checkmark$ & \\
\hline Mascarenhas et al. (2019) & Eficiência e sustentabilidade & $\checkmark$ & $\checkmark$ & $\checkmark$ \\
\hline Sola e Mota (2019) & Fatores que influenciam projetos & $\checkmark$ & $\checkmark$ & $\checkmark$ \\
\hline Zanardo et al. (2018) & Indicadores de desempenho energético & $\checkmark$ & & \\
\hline Toniniet al. (2018) & Elaboração de software & $\checkmark$ & & \\
\hline De Macedo, Mota e Sola (2018) & Motores de alta eficiência & $\checkmark$ & & \\
\hline Silva, Mathias e Bajay (2018) & Análise de desempenho & $\checkmark$ & $\checkmark$ & \\
\hline Aguiar, Pontes e Ferreira (2018) & Eficiência de motores & $\checkmark$ & $\checkmark$ & \\
\hline De Lima, Ribeiro e Perez (2018) & Motivos de ineficiência & $\checkmark$ & $\checkmark$ & $\checkmark$ \\
\hline Guedes et al. (2017) & Acionamento de motores & $\checkmark$ & & \\
\hline Lacerda et al. (2017) & Fornecimento em CC & $\checkmark$ & & \\
\hline Da Silva, Méxas e Quelhas (2017) & Fatores que restringem a sustentabilidade & $\checkmark$ & $\checkmark$ & $\checkmark$ \\
\hline Santana e Bajay (2016) & Políticas energéticas & $\checkmark$ & $\checkmark$ & $\checkmark$ \\
\hline Sauer et al., (2015) & Eficiência de motores & $\checkmark$ & & \\
\hline Da Silva et al. (2015) & Previsão de consumo & $\checkmark$ & & \\
\hline
\end{tabular}

Fonte: Elaborado pelos autores (2020).

No que diz respeito à metodologia empregada na execução dos trabalhos, conforme a descrição visualizada na Tab. 2, grande parte das publicações (nove) utilizou ferramentas de simulação para analisar os cenários, com ou sem aplicação de determinadas medidas de eficiência energética. Outro fato relevante é que apenas cinco das publicações estudadas apresentaram alguma demonstração experimental do que foi proposto, e em parte dessa quantidade, os experimentos foram executados em laboratórios, e não necessariamente no ambiente industrial. Dos demais trabalhos, quatro apresentaram análises de medidas de eficiência energética por meio da aplicação de questionários a determinados setores industriais, realizando um tratamento estatístico das informações coletadas. Somente Santana e Bajay (2016) realizaram um trabalho totalmente de revisão bibliográfica. 
Tabela 2 - Metodologia aplicada aos trabalhos selecionados.

\begin{tabular}{|c|c|c|c|c|}
\hline Publicação & Revisão bibliográfica & Questionário & Simulação & Experimental \\
\hline Da Silva, Oliveira e Souza (2019) & & & $\checkmark$ & \\
\hline Dos Santos e Spinelli (2019) & & & $\checkmark$ & \\
\hline Pontes, Yamauchie Silva (2019) & & & $\checkmark$ & \\
\hline Moraes, Machado e Silva (2019) & & & & $\checkmark$ \\
\hline Birru, Erlich e Martin (2019) & & & & $\checkmark$ \\
\hline Mascarenhas et al. (2019) & & & $\checkmark$ & \\
\hline Sola e Mota (2019) & & $\checkmark$ & & \\
\hline Zanardo et al. (2018) & & & $\checkmark$ & \\
\hline Toniniet al. (2018) & & & $\checkmark$ & \\
\hline De Macedo, Mota e Sola (2018) & & & $\checkmark$ & \\
\hline Silva, Mathias e Bajay (2018) & $\checkmark$ & $\checkmark$ & & \\
\hline Aguiar, Pontes e Ferreira (2018) & & & & $\checkmark$ \\
\hline De Lima, Ribeiro e Perez (2018) & & $\checkmark$ & & \\
\hline Guedes et al. (2017) & & & & $\checkmark$ \\
\hline Lacerda et al. (2017) & & & $\checkmark$ & \\
\hline Da Silva, Méxas e Quelhas (2017) & $\checkmark$ & $\checkmark$ & & \\
\hline Santana e Bajay (2016) & $\checkmark$ & & & \\
\hline Saueret al., (2015) & & & & $\checkmark$ \\
\hline Da Silva et al. (2015) & & & $\checkmark$ & \\
\hline
\end{tabular}

Fonte: Elaborado pelos autores, 2020.

De acordo com a síntese apresentada na Tab. 3, nota-se que os estudos sobre eficiência em motores elétricos estão presentes em oito das publicações selecionadas. Destas, cinco tratam de ações específicas para motores, tais como: padrões de eficiência e regulamentação, sistemas de acionamento, rebobinamento e aplicação em sistemas de bombeamento. Os outros três trabalhos contemplam a análise de motores dentro de projetos mais abrangentes.Destaca-se, também, que seis das publicações tratam do uso de fontes de energias renováveis e complementares, aliadas a projetos de eficiência energética. Destes, somente Tonini et al. (2018) e Lacerda et al. (2017)abordaram aplicações práticas desse tipo de projeto, o restante tratou do tema através revisão de literatura ou aplicação de questionários a diferentes indústrias. Cinco dos trabalhos selecionados discutem as políticas públicas e as regulamentações aplicadas ao setor industrial, a partir da sua eficiência energética e, em sua maioria, trazendo sugestões de melhorias para essas políticas. Ressaltam-se, também, as áreas de estudo em sistemas de bombeamento, refrigeração e climatização, que foram abordadas em apenas um trabalho. 
Tabela 3 - Temas abordados nas publicações selecionadas.

\begin{tabular}{l|l}
\hline \multicolumn{1}{c|}{ Área abordada } & \multicolumn{1}{c}{ Publicações } \\
\hline Motores & $\begin{array}{l}\text { Da Silva, Oliveira e Souza (2019); Birru, Erlich e Martin (2019); Mascarenhas } \\
\text { et al. (2019); Zanardo et al. (2018); De Macedo, Mota e Sola (2018); Aguiar, } \\
\text { Pontes e Ferreira (2018); Guedes et al. (2017); Saueret al., (2015). }\end{array}$ \\
\hline Ventilação & $\begin{array}{l}\text { Da Silva, Oliveira e Souza (2019); Pontes, Yamauchi e Silva (2019); Silva, } \\
\text { Mathias e Bajay (2018). }\end{array}$ \\
\hline Ar comprimido & $\begin{array}{l}\text { Da Silva, Oliveira e Souza (2019); Moraes, Machado e Silva (2019); } \\
\text { Mascarenhas et al. (2019); Zanardo et al. (2018). }\end{array}$ \\
\hline Bombas & Da Silva, Oliveira e Souza (2019 \\
\hline Refrigeração & Da Silva, Oliveira e Souza (2019) \\
\hline Iluminação & $\begin{array}{l}\text { Da Silva, Oliveira e Souza (2019); Moraes, Machado e Silva (2019); } \\
\text { Zanardo et al. (2018); Aguiar, Pontes e Ferreira (2018). }\end{array}$ \\
\hline Aproveitamento de calor & $\begin{array}{l}\text { Dos Santos e Spinelli (2019); Mascarenhas et al. (2019); Silva, Mathias e } \\
\text { Bajay (2018). }\end{array}$ \\
\hline \multirow{2}{*}{ Energias renováveis } & $\begin{array}{l}\text { Moraes, Machado e Silva (2019); Sola e Mota (2019); Tonini et al. (2018); } \\
\text { De Lima, Ribeiro e Perez (2018); Lacerda et al. (2017); Da Silva, Méxas e } \\
\text { Quelhas (2017). }\end{array}$ \\
\hline Climatização & Zanardo et al. (2018) \\
\hline Contratação de energia/ análise tarifária & Zanardo et al. (2018); Tonini et al. (2018); Aguiar, Pontes e Ferreira (2018). \\
\hline Políticas energéticas/regulamentação & $\begin{array}{l}\text { Sola e Mota (2019); De Lima, Ribeiro e Perez (2018); Da Silva, Méxas e } \\
\text { Quelhas (2017); Santana e Bajay (2016); Saueret al., (2015). }\end{array}$ \\
\hline
\end{tabular}

Fonte: Elaborado pelos autores, 2020.

\section{Conclusão}

A partir deste artigo foi possível buscar na literatura especializada algumas das medidas implementadas ou publicadas relacionadas à eficiência energética voltada ao setor industrial do Brasil. Por meio da revisão literária, diagnosticou-se que, nos últimos cinco anos, as publicações relacionadas a essa temática vêm aumentando.Acredita-se que esse fato ocorre por conta do contínuo desenvolvimento do setor, que traz com ele um aumento na demanda no uso dos recursos energéticos e,com isso, vem a necessidade de se buscar novas alternativas, que possam melhorar as ações de eficiência energética nas indústrias brasileiras.

Outro fato observado está ligado à quantidade de publicação por setor. Observou-se que a maioria das medidas de eficiência energética apresentadas estavam ligadas a aplicações em gerais, ou seja, medidas que podem ser aplicadas a qualquer tipo de indústria. Esse fato parece demonstrar que, independentemente dos produtos/bens produzidos, é possível realizar a implementação de políticas voltadas ao consumo eficiente, objetivando melhorar a maneira como os recursos energéticos são utilizados, resultando em reduções de custos no setor.

Verificou-se que uma parcela das publicações não especificava o local ou englobavam várias localidades e regiões.A outra parcela tratou-se de estudos feitos em um único local ou localidades limitadas, sendo que a maior parte foi relativo à região Sudeste, seguida da região Nordeste e da região Centro-Oeste, respectivamente. Observou-se que esse resultado possui forte relação com o apelo industrial de cada região.

Constatou-se, também, que a maior parte dos trabalhos publicados tratava-se de estudos de viabilidade econômica dos projetos. Esse resultado aponta para um aparente despreparo dos pesquisadores na realização de análises econômicas, visto que esse é um aspecto fundamental para qualquer ação de eficiência. Nesse caso, o baixo percentual é esperado, uma vez que, na maioria dos casos, a única consequência ambiental é a redução no consumo de eletricidade, otimizando os recursos energéticos. 
Percebeu-se que a maior parte dos trabalhos avaliaram os aspectos de eficiência energética voltadas aos motores elétricos e, a outra parte, ao uso de fontes de energias renováveis. Acredita-se que esses resultados estão ligados ao fatode que a maior parte doconsumo de energia elétrica nas indústrias é no campo dos acionamentos industriais, que usam motores elétricos e, também, ao contínuo desenvolvimento nas aplicações que utilizam fontes de energias renováveis. Ultimamente, o governo brasileiro tem tido diversas iniciativas voltadas ao incentivo da mini e microgeração de energia elétrica proveniente de fontes renováveis. Entre as vantagens do uso das energias renováveis na mini e microgeração, estão: a compensação de créditos nas faturas de energia elétrica por meio da injeção da energia elétrica gerada excedente nas redes das concessionárias de energia elétrica, além do fato de contribuírem para a diversificação da matriz energética nacional e a diminuição dos impactos ambientais provocados pelas fontes não renováveis.

Ficou evidente, portanto, que existem ações destinadas a melhorar a eficiência energética no setor industrial, sendo esse um ponto de partida importante para que o Brasil possa melhorar cada vez mais as ações e as políticas voltadas à eficiência energéticas nesse setor, tendo em vista que as indústrias são responsáveis pela maior parte do consumo energético do país.

\section{Referências}

AGUIAR, V. P. B.; PONTES, R. S. T.; FERREIRA F. J. T. E. Technical and economic evaluation of efficiency improvement after rewinding in low-power induction motors: abrazilian case. Energies, [S. I.], v. 11, n. 7, p. 1-18, 2018.

BARROS, Benjamim Ferreira; BORELLI, Reinaldo; GEDRA, Ricardo Luís. Eficiência energética: técnicas de aproveitamento, gestão de recursos e fundamentos. São Paulo: Saraiva, 2015.

BIRRU, E.; ERLICH, C.; MARTIN, A. Energy performance comparisons and enhancements in the sugar cane industry. Biomass ConversionandBiorefinery, [S. I.], v. 9, n. 2, p. 267-282, 2019.

CAMIOTO, Flávia de Castro; REBELATTO, Daisy Aparecida do Nascimento; ROCHA, Roberta Teixeira. Análise da eficiência energética nos países do BRICS: um estudo envolvendo a Análise por Envoltória de Dados. Gestão \& Produção, [S. I.], v. 23, n. 1, p. 192-203, 2016.

EMPRESA DE PESQUISA ENERGÉTICA. Matriz energética e elétrica: 2019. Disponível em: http://www. epe.gov.br/pt/abcdenergia/matriz-energetica-e-eletrica. Acesso em: 18 mai. 2020.

FORTES, Márcio Zamboti et al. Análise da adoção de medidores inteligentes como instrumento da política pública de eficiência energética. Engevista, [S. I.], v. 19, n. 2, p. 316-327, 2017.

FLUXO CONSULTORIA. 11 Motivos para realizar um projeto de eficiência energética para indústrias: 2017. Disponível em: https://fluxoconsultoria.poli.ufrj.br/blog/arquitetura-construcao/eficiencia-energeticapara-industrias/. Acesso em: 17 mar. 2020.

GOLDEMBERG, J.; LUCON, N.O. Energias renováveis: um futuro sustentável. Revista USP, São Paulo, v. 72 , p. 6-15, 2007

GUEDES, A. R.; FERREIRA, J. S.; LIMA, A. D. S.; FREITAS, A. A. A.; ANTUNES, F. L. M.; SÁ, E. M. DC bus, an attractive opportunity for energy efficiency in industry. In: INTERNATIONAL SYMPOSIUM ON POWER ELECTRONICS FOR DISTRIBUTED GENERATION SYSTEMs, 8., 2017, Florianópolis. Proceedings [...]. Florianopolis: IEEE, 2017. p. 1-5.

LACERDA, F. A. G.; GOMES, R. A. M.; PORTO, M. P.; CARDOSO, B. J. Directly feeding large dc loads through PV power plants: A new concept applied to industry. In: INDUSTRY APPLICATIONS SOCIETY ANNUAL MEETING, 2017, Cincinnati. Proceedings [...].Cincinnati: IEEE, 2017. p. 1-7. 
LIMA, L. P. D.; RIBEIRO, G. B. de D.; PEREZ, R. The energy mix and energy efficiency analysis for Brazilian dairy industry. Journal of Cleaner Production, [S. I.], v. 181, p. 209-216, 2018

MACEDO, P. P. D.; MOTA, C. M. de M.; SOLA A. V. H. Meeting the Brazilian Energy Efficiency Law: A flexible and interactive multicriteria proposal to replace non-efficient Motors. SustainaibleCities Society, [S. I.], v. 41, p. 822-832, Oct. 2018.

MASCARENHAS, J. dos S.; CHOWDHURY, H.; THIRUGNANASAMBANDAM, M.; CHOWDHURY, T.; SAIDUR, R. Energy, exergy, sustainability, and emission analysis of industrial air compressors. Journal of Cleaner Production, [S. I.], v. 231, p. 183-195, 2019.

MORAES, G. D. O.; MACHADO, R. I.; SILVA, E. J. Energy efficiency study through out the industrial operations of a multinational household appliances manufacturer company in Brazil. Procedia CIRP, [S. I.], v. 80 , p. 228-232, 2019.

PONTES, R. F. F.; YAMAUCHI, W. M.; SILVA, E. K. G. Analysis of the effect of seasonal climate changes on cooling tower efficiency, and strategies for reducing cooling tower Power consumption. Applied Thermal Engineering, [S. I.], v. 161, p. 114148, July 2019.

SANTANA, P. H. De M.; BAJAY, S. V. New approaches for improving energy efficiency in the brazilian industry. Energy Reports, [S. I.], v. 2, p. 62-66, 2016.

SANTOS, R. F. M. D.; SPINELLI, J. E. Análise da geração de energia elétrica por meio da utilização de conversores do tipo TEG em lingotamento contínuo de aços. Revista Matéria, Rio Janeiro, v. 24, n. 3, p. $1-7,2019$.

SAUER, I. L.; TATIZAWA, H.; SALOTTI, F. A. M.; MERCEDES, S. S. A comparative assessment of Brazilian electric motors performance with minimum efficiency Standards. Renewable and SustainableEnergy Reviews, v. 41, p. 308-318, Jan. 2015.

SILVA, R. R. D.; MATHIAS, F. R. de C.; BAJAY, S. V. Potential energy efficiency improvements for the Brazilian iron and steel industry: Fuel and electricity conservation supply curves for integrated steel Mills. Energy, [S. I.], v. 153, p. 816-824, 2018.

SILVA, A. C. D.; MÉXAS, M. P.; QUELHAS, O. L. G. Restrictive factors in implementation of clean technologies in red ceramic industries, Journal OF CleanER, [S. I.], v. 168, p. 441-451, 2017

SILVA, F. L. C. D.; OLIVEIRA, F. L. C.; SOUZA, R. C. A bottom-upbayesianextension for long term electricity consumption forecasting. Energy, [S. I.], v. 167, p. 198-210, 2019.

SILVA. F. L. C. D.; SOUZA, R. C.; OLIVEIRA, F. L. C.; LOURENÇO, P. M.; FAGUNDES, W. D. C. Forecast of long-term electricity consumption of the industrial sub-sector of pulp and paper in Brazil using a bottom-up approach. Procedia Computer Science, [S. I.], v. 55, p. 514-522, 2015.

SOLA, Antonio Vanderley Herrero; KOVALESKI, João Luiz. Eficiência energética nas indústrias: cenários \& oportunidades.In: ENCONTRO NACIONAL DE ENGENHARIA DA PRODUÇÃO, 24., 2004, Florianópolis. Anais [...]. Florianópolis, v. 14, 2004. p. 1-8.

SOLA, A. V. H.; MOTA, C. M. M. Influencing factors on energy management in industries. Journal of Cleaner Production, [S. I.], v. 248, 2019.

TONINI, M. S. P.; ROCHA, H. R. O.; SILVESTRE, L. J.; CELESTE, W. C.; COURA, D. J. C.; FIOROTTI, R. Industrial demand management consideringdistributedgenerationanddifferentiated rates in time. In: BRAZILIAN ELECTRICAL SYSTEMS SYMPOSIUM, 7., 2018, Niterói. Proceedings [...]. Niterói: IEEE, 2018. p. 1-6. 
ZANARDO, R. P.; SILUK, J. C. M.; SAVIAN, F. de S.; SCHNEIDER, P. S. Energy audit model based on a performance evaluation system. Energy, [S. I.], v. 154, p. 544-552, 2018.

\section{Sobre os autores}

\section{Francisca Lívia Costa Pires}

Mestranda em Energias Renováveis pelo Instituto Federal de Educação, Ciência e Tecnologia do Ceará (IFCE). Aluna de Especialização em Eficiência Energética pela Universidade de Fortaleza (Unifor). Graduada em Engenharia Elétrica pela Universidade Federal do Ceará (UFC) (2007). Formação técnica em Telecomunicações pelo Centro Federal de Educação Tecnológica (CEFET-CE) (2002). Professora do ensino básico, técnico e tecnológico e coordenadora de ensino no Instituto Federal de Educação, Ciência e Tecnologia do Ceará (IFCE), campus Pecém.

\section{Raylla Silveira Souto}

Mestranda em Física Aplica pela Universidade Estadual do Ceará (Uece). Aluna de Especialização em Eficiência Energética pela Universidade de Fortaleza (Unifor). Graduada em Engenharia Elétrica pela Universidade de Fortaleza (Unifor) (2017).

\section{Paulo Henrique Pereira Silva}

Bacharel em Física pela Universidade Estadual do Ceará (Uece), mestre em Engenharia Nuclear pelo Instituto Militar de Engenharia (IME) e doutor em Engenharia Elétrica pela Universidade Federal do Ceará (UFC). É professor efetivo do curso Tecnológico em Energias Renováveis da Universidade de Fortaleza (Unifor). É integrante da Cooperativa Energias Renováveis do Nordeste (Coodensol). Possui interesse na área de energias alternativas e suas aplicações, com ênfase em energia eólica, solar, nuclear e eficiência energética, estudando, principalmente, os seguintes temas: gerador de indução duplamente alimentado (DFIG), qualidade de energia, equipamentos FACTS, reator nuclear de espectro rápido, estudo de matrizes energéticas e conservação de energia.

Recebido em: 22.06.2020

Aceito em: 20.07.2020 